\title{
PELABELAN PRIME CORDIAL UNTUK GRAF BUKU DAN GRAF MATAHARI YANG DIPERUMUM
}

\author{
S.Pranata1 , I. W. Sudarsana ${ }^{2}$ dan S.Musdalifah ${ }^{3}$ \\ 1,2,3Program Studi Matematika Jurusan Matematika FMIPA Universitas Tadulako \\ Jalan Soekarno-Hatta Km. 09 Tondo, Palu 94118, Indonesia. \\ 1supitpranata@gmail.com, 2sudarsanaiwayan@yahoo.co.id, 3selvymusdalifah@yahoo.com
}

\section{ABSTRACT}

A prime cordial labeling of a graph $G$ with the vertex set $V(G)$ is a bijection $f: V(G) \rightarrow\{1,2,3, \cdots, p\}$, where $p$ is the number of vertex in the graph $\mathrm{G}$ and the induced $\mathrm{f}^{*} \mathrm{E}(\mathrm{G}) \rightarrow\{0,1\}$ is defined by

$$
f^{*}(e=u v)=\left\{\begin{array}{l}
1 ; \text { if } \operatorname{gcd}(f(u), f(v))=1, \\
0 ; \text { otherwise. }
\end{array}\right.
$$

satisfies the condition $\left|\mathrm{e}_{\mathrm{f}^{*}}(0)-\mathrm{e}_{\mathrm{f}^{*}}(1)\right| \leq 1$, where $\mathrm{e}_{\mathrm{f}^{*}}(\mathrm{i})$ is the number of edges having label $\mathrm{i}=0$ and 1 . A graph that contains prime cordial labeling is called prime cordial graph. The results of the study showed that book graph $K_{1, n} \times P_{m}$ for $m \in\{3,4,5,6,7\}$ and sun graph $C_{n} \odot \overline{K_{m}}$ for $m \in\{2,3,5\}$ satisfy prime cordial labeling.

Keywords : Book Graph, Prime Cordial Labeling, Sun Graph

\section{ABSTRAK}

Pelabelan prime cordial dari suatu graf $G$ dengan himpunan titik $V(G)$ adalah bijeksi $f: V(G) \rightarrow\{1,2,3, \cdots, p\}$, dimana $p$ adalah banyaknya titik di graf $G$ dan fungsi induksi $f^{*}: E(G) \rightarrow\{0,1\}$ yang didefinisikan oleh

$$
\mathrm{f}^{*}(\mathrm{e}=\mathrm{uv})=\left\{\begin{array}{l}
1 ; \text { jika gcd }(\mathrm{f}(\mathrm{u}), \mathrm{f}(\mathrm{v}))=1, \\
0 ; \text { lainnya. }
\end{array}\right.
$$

dan memenuhi syarat $\left|\mathrm{e}_{\mathrm{f}^{*}}(0)-\mathrm{e}_{\mathrm{f}^{*}}(1)\right| \leq 1$, dimana $\mathrm{e}_{\mathrm{f}^{*}}(\mathrm{i})$ adalah banyaknya sisi yang mempunyai labell $\mathrm{i}=0$ dan 1. Sebuah graf yang memuat pelabelan prime cordial disebut graf prime cordial. Hasil penelitian menunjukkan bahwa graf buku $\mathrm{K}_{1, \mathrm{n}} \times \mathrm{P}_{\mathrm{m}}$ untuk $\mathrm{m} \in\{3,4,5,6,7\}$ dan graf matahari $\mathrm{C}_{\mathrm{n}} \odot \overline{\mathrm{K}_{\mathrm{m}}}$ untuk $\mathrm{m} \in\{2,3,5\}$ memenuhi pelabelan prime cordial.

Kata Kunci: Graf Buku, Graf Matahari, Pelabelan Prime Cordial 


\section{PENDAHULUAN}

\subsection{Latar Belakang}

Lahirnya teori graf pertama kali diperkenalkan oleh Leonhard Euler seorang matematikawan berkebangsaan Swiss pada tahun 1736 melalui tulisan Euler yang berisi tentang upaya pemecahan masalah jembatan Konigsberg yang sangat terkenal di Eropa. Banyak yang dapat dipelajari dari suatu graf, salah satu di antaranya adalah mengenai pelabelan graf.

Pelabelan graf adalah suatu pemberian nilai (dengan bilangan bulat positif) pada titik atau sisi dari graf atau keduanya sehingga memenuhi kondisi tertentu. Bilangan-bilangan tersebut disebut label. Jika yang diberi label hanya titik (vertex) saja, maka pelabelannya disebut pelabelan titik (vertex). Jika yang diberi label hanya sisi (edge) saja, maka pelabelannya disebut pelabelan sisi (edge). Sedangkan jika keduanya, titik dan sisi diberi label, maka pelabelannya disebut pelabelan total (Cahayani,dkk., 2013).

Pelabelan prime cordial merupakan suatu bentuk pelabelan pada titik yang label sisinya mengikuti (induced) label titiknya, yang didefinisikan sebagai $f: V(G) \rightarrow\{1,2,3, \cdots, p\}$, dimana $\mathrm{p}$ adalah banyaknya titik di graf $\mathrm{G}$ dengan sifat $\mathrm{f}^{*}(\mathrm{e}=\mathrm{uv})=1$ jika $\operatorname{gcd}(\mathrm{f}(\mathrm{u}), \mathrm{f}(\mathrm{v}))=1$ dan $\mathrm{f}^{*}(\mathrm{e}=\mathrm{uv})=0$ untuk yang lainnya, dan memenuhi $\left|\mathrm{e}_{\mathrm{f}^{*}}(0)-\mathrm{e}_{\mathrm{f}^{*}}(1)\right| \leq 1$.

Kajian graf terkait pelabelan prime cordial antara lain Sundaram, dkk (2005) yang mengkaji graf siklus $C_{n}$ (untuk $n \geq 6$ ), graf lintasan $P_{n}$ (untuk $n \neq 3$ atau 5), graf naga, graf mahkota dan graf tangga, Babujee dan Shobana (2009) yang mengkaji graf matahari $C_{n} \odot$ $K_{1}$, Vaidya dan Vihol (2010) yang mengkaji graf total lintasan $T\left(P_{n}\right)$ (untuk $n \geq 5$ ), graf total siklus $T\left(C_{n}\right)$ (untuk $n \geq 5$ ), dan komposisi $P_{2}\left(P_{m}\right)$ (untuk $m \geq 5$ ), serta Rohman (2011) yang mengkaji graf buku $\mathrm{K}_{1, \mathrm{n}} \times \mathrm{P}_{2}$.

\subsection{Rumusan Masalah}

Permasalahan yang akan dibahas dalam penelitian ini adalah bagaimana formula pelabelan prime cordial untuk graf buku dan graf matahari yang diperumum.

\subsection{Tujuan Penelitian}

Tujuan dari penelitian ini adalah untuk mendapatkan formula pelabelan prime cordial pada graf buku dan graf matahari yang diperumum. 


\subsection{Batasan Masalah}

Penelitian ini terbatas pada dua graf berikut :

1. Graf buku diperumum $\left(K_{1, n} \times P_{m}\right)$ untuk $m \in\{3,4,5,6,7\}$, dimana :

1) untuk $m=3,4$ maka $n \geq 3$;

2) untuk $m=5$, maka $n \geq 6$;

3) untuk $m=6$, maka $n \geq 10$;

4) untuk $\mathrm{m}=7$, maka $\mathrm{n} \geq 18$;

2. Graf matahari diperumum $\left(C_{n} \odot \overline{K_{m}}\right)$ untuk $n \geq 3$ dan $m \in\{2,3,5\}$.

\subsection{Manfaat Penelitian}

Manfaat dari penelitian ini adalah sebagai berikut :

1. Sebagai referensi dan tambahan ilmu pengetahuan dalam mengembangkan penelitianpenelitian di bidang teori graf, khususnya tentang pelabelan prime cordial.

2. Untuk mengaplikasikan dan mengembangkan ilmu yang selama ini menjadi bidang minat yang dipelajari.

\section{METODE PENELITIAN}

Adapun prosedur yang dilakukan pada penelitian ini adalah sebagai berikut :

1. Memulai penelitian

2. Melakukan studi literatur

3. Menotasikan titik dan sisi pada graf buku dan graf matahari yang diperumum

4. Melabeli titik dan sisi pada graf sesuai dengan syarat pelabelan prime cordial

5. Menganalisa pola untuk merumuskan pelabelan prime cordial

6. Membuat formula pelabelan prime cordial

7. Membuktikan formula pelabelan untuk setiap titik. Jika terbukti maka diperoleh hasil penelitian. Jika tidak terbukti maka kembali ke langkah 4 yaitu melabeli titik dan sisi pada graf sesuai dengan syarat pelabelan prime cordial

8. Memperoleh hasil

9. Kesimpulan

10. Selesai

\section{HASIL DAN PEMBAHASAN}

Pada bagian ini akan dibahas mengenai pelabelan prime cordial pada graf buku $\left(\mathrm{K}_{1, \mathrm{n}} \times\right.$ $\mathrm{P}_{3}, \mathrm{~K}_{1, \mathrm{n}} \times \mathrm{P}_{4}, \mathrm{~K}_{1, \mathrm{n}} \times \mathrm{P}_{5}, \mathrm{~K}_{1, \mathrm{n}} \times \mathrm{P}_{6}$ dan $\left.\mathrm{K}_{1, \mathrm{n}} \times \mathrm{P}_{7}\right)$ dan graf matahari $\left(\mathrm{C}_{\mathrm{n}} \odot \overline{\mathrm{K}_{2}}, \mathrm{C}_{\mathrm{n}} \odot \overline{\mathrm{K}_{3}}\right.$ dan $\left.\mathrm{C}_{\mathrm{n}} \odot \overline{\mathrm{K}_{5}}\right)$ dengan $\mathrm{n}$ bilangan asli. Untuk menunjukkan pelabelan prime cordial pada graf buku dan graf matahari yang diperumum, maka langkah-langkah yang akan dilakukan yaitu menotasikan titik dan sisi pada graf, melabeli graf dan membuat formula dengan menganalisa pola pelabelan. 


\section{Definisi 1 :}

Graf buku diperumum adalah graf yang diperoleh dari hasil perkalian graf bintang $\mathrm{K}_{1, \mathrm{n}}$ dan graf lintasan $\mathrm{P}_{\mathrm{m}}$, atau dapat ditulis sebagai $\mathrm{K}_{1, \mathrm{n}} \times \mathrm{P}_{\mathrm{m}}$. Penotasian graf buku $\mathrm{K}_{1, \mathrm{n}} \times \mathrm{P}_{\mathrm{m}}$ tersaji dalam Gambar 1.

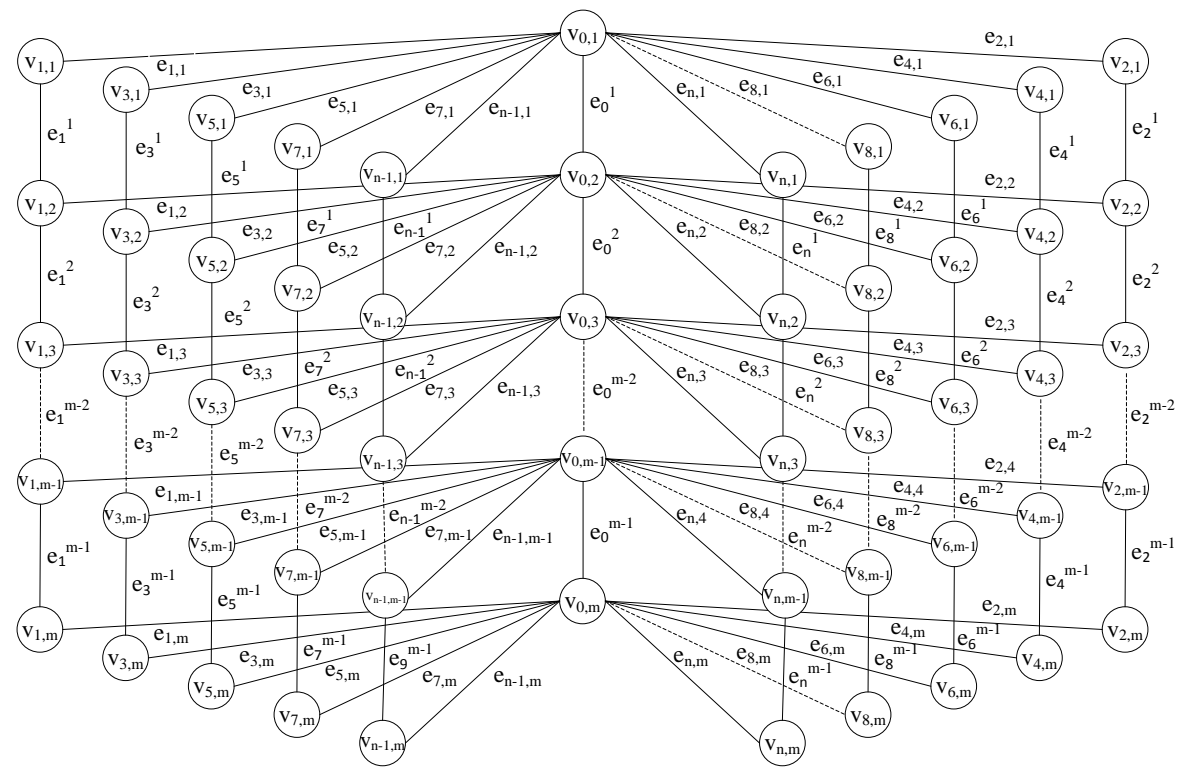

Gambar 1 : Penotasian titik dan sisi pada graf buku $\mathrm{K}_{1, \mathrm{n}} \times \mathrm{P}_{\mathrm{m}}$

Berdasarkan Gambar 1, maka graf buku $\mathrm{K}_{1, \mathrm{n}} \times \mathrm{P}_{\mathrm{m}}$ dapat dinotasikan sebagai berikut : Himpunan titik

$V\left(K_{1, n} \times P_{m}\right)=\left\{v_{i, j} \mid i=0,1, \ldots, n ; j=1,2, \ldots, m\right\}$

Himpunan sisi

$\mathrm{E}\left(\mathrm{K}_{1, \mathrm{n}} \times \mathrm{P}_{\mathrm{m}}\right)=\left\{\mathrm{e}_{\mathrm{i}}^{\mathrm{t}}=\mathrm{v}_{\mathrm{i}, \mathrm{t}} \mathrm{v}_{\mathrm{i}, \mathrm{t}+1}|\mathrm{i}=0,1, \ldots, \mathrm{n}| \mathrm{t}=1,2, \ldots, \mathrm{m}-1\right\}$

$u\left\{e_{i, j}=v_{0, j} v_{i, j} \mid i=1,2, \ldots, n ; j=1,2, \ldots, m\right\}$

Graf buku $K_{1, n} \times P_{m}$ mempunyai $m(n+1)$ titik dan $(2 m-1) n+(m-1)$ sisi

Selanjutnya pada penelitian ini akan dibahas graf buku $K_{1, n} \times P_{m}$ untuk $m \in\{3,4,5,6,7\}$.

\section{Definisi 2 :}

Graf matahari diperumum adalah graf yang dibentuk dari graf siklus $\mathrm{C}_{\mathrm{n}}$ korona komplemen graf lengkap $\overline{K_{m}}$, atau dapat ditulis sebagai $C_{n} \odot \overline{K_{m}}$. Derajat titik pada graf siklus $C_{n}$ akan menjadi $m+$ 2, sedangkan derajat titik lainnya adalah 1 . Penotasian graf matahari $C_{n} \odot \overline{K_{m}}$ tersaji dalam Gambar 2. 


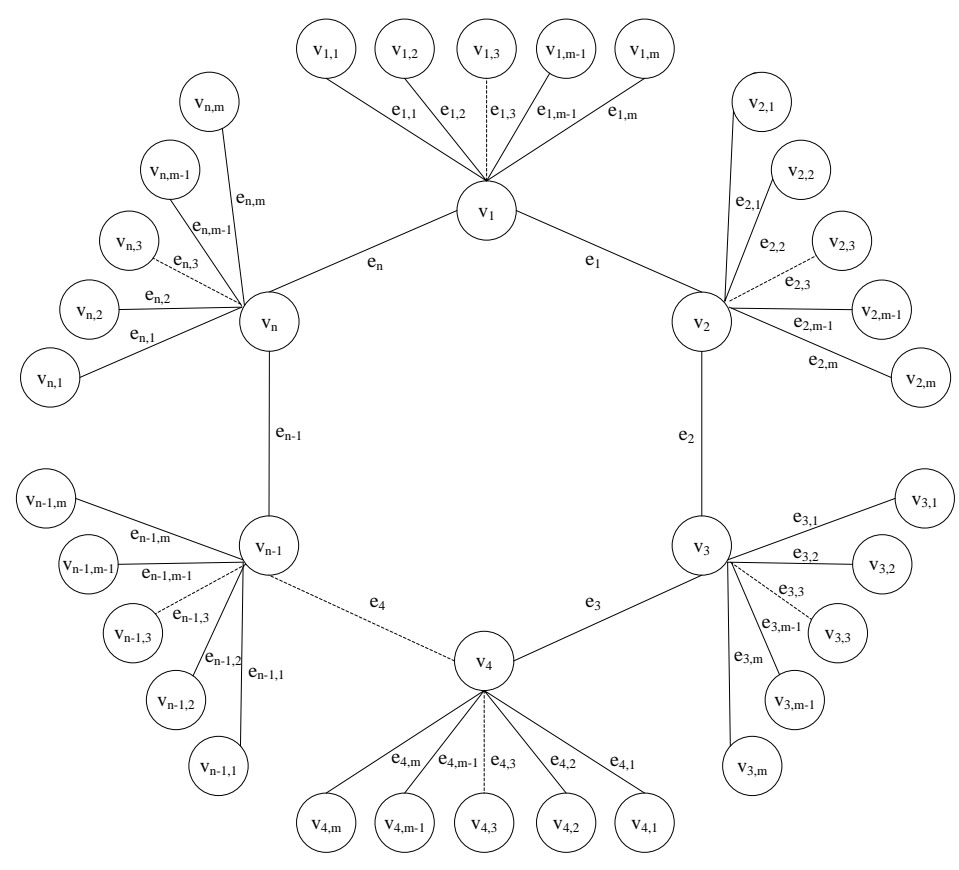

Gambar 2 : Penotasian titik dan sisi pada graf matahari $\mathrm{C}_{\mathrm{n}} \odot \overline{\mathrm{K}_{\mathrm{m}}}$

Berdasarkan Gambar 2, maka graf matahari $\mathrm{C}_{\mathrm{n}} \odot \overline{\mathrm{K}_{\mathrm{m}}}$ dapat dinotasikan sebagai berikut : Himpunan titik

$\mathrm{V}\left(\mathrm{C}_{\mathrm{n}} \odot \overline{\mathrm{K}_{\mathrm{m}}}\right)=\left\{\mathrm{v}_{\mathrm{i}} \mid \mathrm{i}=1,2, \ldots, \mathrm{n}\right\} \cup\left\{\mathrm{v}_{\mathrm{i}, \mathrm{j}} \mid \mathrm{i}=1,2, \ldots, \mathrm{n} ; \mathrm{j}=1,2, \ldots, \mathrm{m}\right\}$

Himpunan sisi

$\mathrm{E}\left(\mathrm{C}_{\mathrm{n}} \odot \overline{\mathrm{K}_{\mathrm{m}}}\right)=\left\{\mathrm{e}_{\mathrm{i}}=\mathrm{v}_{\mathrm{i}} \mathrm{v}_{\mathrm{i}+1}, \mathrm{e}_{\mathrm{n}}=\mathrm{v}_{1} \mathrm{v}_{\mathrm{n}} \mid \mathrm{i}=1,2, \ldots, \mathrm{n}-1\right\} \cup\left\{\mathrm{e}_{\mathrm{i}, \mathrm{j}}=\mathrm{v}_{\mathrm{i}} \mathrm{v}_{\mathrm{i}, \mathrm{j}}\right.$ $\mid i=1,2, \ldots, n ; j=1,2, \ldots, m\}$

Graf matahari $\mathrm{C}_{\mathrm{n}} \odot \overline{\mathrm{K}_{\mathrm{m}}}$ mempunyai $\mathrm{n}(\mathrm{m}+1)$ titik dan sisi

Selanjutnya pada penelitian ini akan dibahas graf matahari $C_{n} \odot \overline{K_{m}}$ untuk $m \in\{2,3,5\}$.

\section{Teorema 1 :}

Graf buku $\mathrm{K}_{1, \mathrm{n}} \times \mathrm{P}_{\mathrm{m}}$ adalah prime cordial untuk $\mathrm{m} \in\{3,4,5,6,7\}$, dimana :

1) untuk $m=3,4$ maka $n \geq 3$;

2) untuk $m=5$, maka $n \geq 6$;

3) untuk $m=6$, maka $n \geq 10$;

4) untuk $\mathrm{m}=7$, maka $\mathrm{n} \geq 18$; 


\section{Bukti :}

Kasus $\mathrm{K}_{1, \mathbf{n}} \times \mathbf{P}_{3}$ :

\section{Subkasus $1:$ n ganjil}

Akan ditunjukkan bahwa graf buku $\mathrm{K}_{1, \mathrm{n}} \times \mathrm{P}_{3}$ untuk $\mathrm{n} \geq 3$ adalah prime cordial dalam bentuk fungsi sebagai berikut:

$$
\begin{aligned}
& \mathrm{f}_{1}\left(\mathrm{v}_{\mathrm{i}, 1}\right)=\left\{\begin{array}{cl}
2 & ; \mathrm{i}=0 \\
\mathrm{i}^{2} & ; \mathrm{i}=1,2 \\
3 \mathrm{i}+1 & ; 3 \leq \mathrm{i} \leq \mathrm{n} \text { (i ganjil) } \\
3 \mathrm{i}-1 & ; 4 \leq \mathrm{i} \leq \mathrm{n}-1 \text { (i genap) }
\end{array}\right. \\
& \mathrm{f}_{1}\left(\mathrm{v}_{\mathrm{i}, 2}\right)=\left\{\begin{array}{cl}
6 & ; \mathrm{i}=0 \\
3 \mathrm{i}+2 & ; \mathrm{i}=1,2 \\
3 \mathrm{i}+3 & ; 3 \leq \mathrm{i} \leq \mathrm{n}-2 \text { (i ganjil) } \\
3 \mathrm{i}+1 & ; 4 \leq \mathrm{i} \leq \mathrm{n}-1 \text { (i genap) } \\
3 \mathrm{i}+2 & ; \mathrm{i}=\mathrm{n}
\end{array}\right. \\
& \mathrm{f}_{1}\left(\mathrm{v}_{\mathrm{i}, 3}\right)= \begin{cases}3 & ; \mathrm{i}=0 \\
2 \mathrm{i}+5 & ; \mathrm{i}=1,2 \\
3 \mathrm{i}+5 & ; 3 \leq \mathrm{i} \leq \mathrm{n}-2 \text { (i ganjil) } \\
3 \mathrm{i}+3 & ; 4 \leq \mathrm{i} \leq \mathrm{n}-1 \text { (i genap) } \\
3 \mathrm{i}+3 & ; \mathrm{i}=\mathrm{n}\end{cases}
\end{aligned}
$$

\section{Subkasus 2 : n genap}

Akan ditunjukkan bahwa graf buku $\mathrm{K}_{1, \mathrm{n}} \times \mathrm{P}_{3}$ untuk $\mathrm{n} \geq 4$ adalah prime cordial dalam bentuk fungsi sebagai berikut :

$$
\begin{aligned}
& \mathrm{f}_{2}\left(\mathrm{v}_{\mathrm{i}, 1}\right)=\left\{\begin{array}{cl}
2 & ; \mathrm{i}=0 \\
\mathrm{i}^{2} & ; \mathrm{i}=1,2 \\
3 \mathrm{i}+1 & ; 3 \leq \mathrm{i} \leq \mathrm{n}-1 \text { (i ganjil) } \\
3 \mathrm{i}-1 & ; 4 \leq \mathrm{i} \leq \mathrm{n} \text { (i genap) }
\end{array}\right. \\
& \mathrm{f}_{2}\left(\mathrm{v}_{\mathrm{i}, 2}\right)=\left\{\begin{array}{cl}
6 & ; \mathrm{i}=0 \\
3 \mathrm{i}+2 & ; \mathrm{i}=1,2 \\
3 \mathrm{i}+3 & ; 3 \leq \mathrm{i} \leq \mathrm{n}-1 \text { (i ganjil) } \\
3 \mathrm{i}+1 & ; 4 \leq \mathrm{i} \leq \mathrm{n} \text { (i genap) }
\end{array}\right. \\
& \mathrm{f}_{2}\left(\mathrm{v}_{\mathrm{i}, 3}\right)=\left\{\begin{array}{cc}
3 & ; \mathrm{i}=0 \\
2 \mathrm{i}+5 & ; \mathrm{i}=1,2 \\
3 \mathrm{i}+5 & ; 3 \leq \mathrm{i} \leq \mathrm{n}-1 \text { (i ganjil) } \\
3 \mathrm{i}+3 & ; 4 \leq \mathrm{i} \leq \mathrm{n} \text { (i genap) }
\end{array}\right.
\end{aligned}
$$

Kasus $\mathrm{K}_{1, \mathrm{n}} \times \mathbf{P}_{\mathbf{4}}$ :

\section{Subkasus $1:$ n ganjil}

Akan ditunjukkan bahwa graf buku $\mathrm{K}_{1, \mathrm{n}} \times \mathrm{P}_{4}$ untuk $\mathrm{n} \geq 3$ adalah prime cordial dalam bentuk fungsi sebagai berikut :

$$
\begin{aligned}
& \mathrm{f}_{1}\left(\mathrm{v}_{\mathrm{i}, 1}\right)=\left\{\begin{array}{cl}
2-\mathrm{i} & ; \mathrm{i}=0,1 \\
\mathrm{i}+3 & ; \mathrm{i}=2,3 \\
4 \mathrm{i}+1 & ; 4 \leq \mathrm{i} \leq \mathrm{n}-1 \text { (i genap) } \\
4 \mathrm{i}-2 & ; 5 \leq \mathrm{i} \leq \mathrm{n} \text { (i ganjil) }
\end{array}\right. \\
& \mathrm{f}_{1}\left(\mathrm{v}_{\mathrm{i}, 2}\right)=\left\{\begin{array}{cl}
4-\mathrm{i} & ; \mathrm{i}=0,1 \\
3 \mathrm{i}+1 & ; \mathrm{i}=2,3 \\
4 \mathrm{i}+3 & ; 4 \leq \mathrm{i} \leq \mathrm{n}-1 \text { (i genap) } \\
4 \mathrm{i} & ; 5 \leq \mathrm{i} \leq \mathrm{n} \text { (i ganjil) }
\end{array}\right.
\end{aligned}
$$




$$
\begin{aligned}
& \mathrm{f}_{1}\left(\mathrm{v}_{\mathrm{i}, 3}\right)= \begin{cases}\mathrm{i}+8 & ; \mathrm{i}=0,1 \\
\mathrm{i}+9 & ; \mathrm{i}=2,3 \\
4 \mathrm{i}+5 & ; 4 \leq \mathrm{i} \leq \mathrm{n}-1 \text { (i genap) } \\
4 \mathrm{i}+2 & ; 5 \leq \mathrm{i} \leq \mathrm{n} \text { (i ganjil) }\end{cases} \\
& \mathrm{f}_{1}\left(\mathrm{v}_{\mathrm{i}, 4}\right)= \begin{cases}16-\mathrm{i} & ; \mathrm{i}=0,1 \\
\mathrm{i}+11 & ; \mathrm{i}=2,3 \\
4 \mathrm{i}+7 & ; 4 \leq \mathrm{i} \leq \mathrm{n}-1 \text { (i genap) } \\
4 \mathrm{i}+4 & ; 5 \leq \mathrm{i} \leq \mathrm{n} \text { (i ganjil) }\end{cases}
\end{aligned}
$$

\section{Subkasus 2 : n genap}

Akan ditunjukkan bahwa graf buku $\mathrm{K}_{1, \mathrm{n}} \times \mathrm{P}_{4}$ untuk $\mathrm{n} \geq 4$ adalah prime cordial dalam bentuk fungsi sebagai berikut :

$$
\begin{aligned}
& \mathrm{f}_{2}\left(\mathrm{v}_{\mathrm{i}, 1}\right)= \begin{cases}2-\mathrm{i} & ; \mathrm{i}=0,1 \\
\mathrm{i}+3 & ; \mathrm{i}=2,3 \\
4 \mathrm{i}+1 & ; 4 \leq \mathrm{i} \leq \mathrm{n} \text { (i genap) } \\
4 \mathrm{i}-2 & ; 5 \leq \mathrm{i} \leq \mathrm{n}-1 \text { (i ganjil) }\end{cases} \\
& \mathrm{f}_{2}\left(\mathrm{v}_{\mathrm{i}, 2}\right)=\left\{\begin{array}{cl}
4-\mathrm{i} & ; \mathrm{i}=0,1 \\
3 \mathrm{i}+1 & ; \mathrm{i}=2,3 \\
4 \mathrm{i}+3 & ; 4 \leq \mathrm{i} \leq \mathrm{n} \text { (i genap) } \\
4 \mathrm{i} & ; 5 \leq \mathrm{i} \leq \mathrm{n}-1 \text { (i ganjil) }
\end{array}\right. \\
& \mathrm{f}_{2}\left(\mathrm{v}_{\mathrm{i}, 3}\right)= \begin{cases}\mathrm{i}+8 & ; \mathrm{i}=0,1 \\
\mathrm{i}+9 & ; \mathrm{i}=2,3 \\
4 \mathrm{i}+5 & ; 4 \leq \mathrm{i} \leq \mathrm{n}-2 \text { (i genap) } \\
4 \mathrm{i}+2 & ; 5 \leq \mathrm{i} \leq \mathrm{n}-1 \text { (i ganjil) } \\
4 \mathrm{i}+4 & ; \mathrm{i}=\mathrm{n}\end{cases} \\
& \mathrm{f}_{2}\left(\mathrm{v}_{\mathrm{i}, 4}\right)= \begin{cases}16-\mathrm{i} & ; \mathrm{i}=0,1 \\
\mathrm{i}+11 & ; \mathrm{i}=2,3 \\
4 \mathrm{i}+7 & ; 4 \leq \mathrm{i} \leq \mathrm{n}-2 \text { (i genap) } \\
4 \mathrm{i}+4 & ; 5 \leq \mathrm{i} \leq \mathrm{n}-1 \text { (i ganjil) } \\
4 \mathrm{i}+2 & ; \mathrm{i}=\mathrm{n}\end{cases}
\end{aligned}
$$

Kasus $\mathrm{K}_{1, \mathrm{n}} \times \mathrm{P}_{\mathbf{5}}$ :

\section{Subkasus 1 : n genap}

Akan ditunjukkan bahwa graf buku $\mathrm{K}_{1, \mathrm{n}} \times \mathrm{P}_{5}$ untuk $\mathrm{n} \geq 6$ adalah prime cordial dalam bentuk fungsi sebagai berikut :

$$
\begin{aligned}
& \mathrm{f}_{1}\left(\mathrm{v}_{\mathrm{i}, 1}\right)=\left\{\begin{array}{cl}
\mathrm{i}+2 & ; \mathrm{i}=0,1 \\
5 \mathrm{i}-9 & ; \mathrm{i}=2,3 \\
4 \mathrm{i}+1 & ; \mathrm{i}=4 \\
5(\mathrm{i}-1) & ; \mathrm{i}=5,7 \\
5 \mathrm{i}+1 & ; 6 \leq \mathrm{i} \leq \mathrm{n} \text { (i genap) } \\
5 \mathrm{i}-3 & ; 9 \leq \mathrm{i} \leq \mathrm{n}-1 \text { (i ganjil) }
\end{array}\right. \\
& \mathrm{f}_{1}\left(\mathrm{v}_{\mathrm{i}, 2}\right)=\left\{\begin{array}{cl}
5 \mathrm{i}+4 & ; \mathrm{i}=0,1 \\
5(\mathrm{i}-1) & ; \mathrm{i}=2,3 \\
3 \mathrm{i}+7 & ; \mathrm{i}=4,5 \\
5 \mathrm{i}+3 & ; 6 \leq \mathrm{i} \leq \mathrm{n} \text { (i genap) } \\
5 \mathrm{i}-1 & ; 7 \leq \mathrm{i} \leq \mathrm{n}-1 \text { ( } \mathrm{i} \text { ganjil) }
\end{array}\right. \\
& \mathrm{f}_{1}\left(\mathrm{v}_{\mathrm{i}, 3}\right)=\left\{\begin{array}{cl}
7 \mathrm{i}+8 & ; \mathrm{i}=0,1 \\
5 \mathrm{i}-3 & ; \mathrm{i}=2,3 \\
\mathrm{i}+19 & ; \mathrm{i}=4,5 \\
5(\mathrm{i}+1) & ; 6 \leq \mathrm{i} \leq \mathrm{n} \text { (i genap) } \\
5 \mathrm{i}+1 & ; 7 \leq \mathrm{i} \leq \mathrm{n}-1 \text { (i ganjil) }
\end{array}\right.
\end{aligned}
$$


$\mathrm{f}_{1}\left(\mathrm{v}_{\mathrm{i}, 4}\right)= \begin{cases}5 \mathrm{i}+16 & ; \mathrm{i}=0,1 \\ 3 \mathrm{i}+5 & ; \mathrm{i}=2,3 \\ \mathrm{i}+21 & ; \mathrm{i}=4,5 \\ 5 \mathrm{i}+7 & ; 6 \leq \mathrm{i} \leq \mathrm{n}-2 \text { (i genap) } \\ 5 \mathrm{i}+3 & ; 7 \leq \mathrm{i} \leq \mathrm{n}-1 \text { (i ganjil) } \\ 5 \mathrm{i}+4 & ; \mathrm{i}=\mathrm{n}\end{cases}$
$\mathrm{f}_{1}\left(\mathrm{v}_{\mathrm{i}, 5}\right)=\left\{\begin{array}{cl}32-5 \mathrm{i} & ; \mathrm{i}=0,1 \\ 5 \mathrm{i}+3 & ; \mathrm{i}=2,3,5 \\ 5 \mathrm{i}+9 & ; 4 \leq \mathrm{i} \leq \mathrm{n}-2 \text { (i genap) } \\ 5(\mathrm{i}+1) & ; 7 \leq \mathrm{i} \leq \mathrm{n}-1 \text { (i ganjil) } \\ 5 \mathrm{i}+2 & ; \mathrm{i}=\mathrm{n}\end{array}\right.$

\section{Subkasus 2 : n ganjil}

Akan ditunjukkan bahwa graf buku $\mathrm{K}_{1, \mathrm{n}} \times \mathrm{P}_{5}$ untuk $\mathrm{n} \geq 7$ adalah prime cordial dalam bentuk fungsi sebagai berikut:

$\mathrm{f}_{2}\left(\mathrm{v}_{\mathrm{i}, 1}\right)=\left\{\begin{array}{cl}2-\mathrm{i} & ; \mathrm{i}=0,1 \\ \mathrm{i}+3 & ; \mathrm{i}=2,3 \\ 5 \mathrm{i}-1 & ; \mathrm{i}=4 \\ 5(\mathrm{i}-1) & ; \mathrm{i}=5,7 \\ 5 \mathrm{i}+1 & ; 6 \leq \mathrm{i} \leq \mathrm{n}-1 \text { (i genap) } \\ 5 \mathrm{i}-3 & ; 9 \leq \mathrm{i} \leq \mathrm{n} \text { (i ganjil) }\end{array}\right.$

$\mathrm{f}_{2}\left(\mathrm{v}_{\mathrm{i}, 2}\right)= \begin{cases}4-\mathrm{i} & ; \mathrm{i}=0,1 \\ 3 \mathrm{i}+1 & ; \mathrm{i}=2.3 \\ 5 \mathrm{i}+3 & ; 4 \leq \mathrm{i} \leq \mathrm{n}-1 \text { (i genap) } \\ 5 \mathrm{i}-3 & ; \mathrm{i}=5 \\ 5 \mathrm{i}-1 & ; 7 \leq \mathrm{i} \leq \mathrm{n} \text { (i ganjil) }\end{cases}$

$\mathrm{f}_{2}\left(\mathrm{v}_{\mathrm{i}, 3}\right)=\left\{\begin{array}{cl}\mathrm{i}+8 & ; \mathrm{i}=0,1 \\ \mathrm{i}+9 & ; \mathrm{i}=2,3 \\ 5(\mathrm{i}+1) & ; 4 \leq \mathrm{i} \leq \mathrm{n}-1 \text { (i genap) } \\ 5 \mathrm{i}-1 & ; \mathrm{i}=5 \\ 5 \mathrm{i}+1 & ; 7 \leq \mathrm{i} \leq \mathrm{n} \text { (i ganjil) }\end{array}\right.$

$\mathrm{f}_{2}\left(\mathrm{v}_{\mathrm{i}, 4}\right)= \begin{cases}16-\mathrm{i} & ; \mathrm{i}=0,1 \\ \mathrm{i}+11 & ; \mathrm{i}=2,3 \\ 5 \mathrm{i}+7 & ; 4 \leq \mathrm{i} \leq \mathrm{n}-1 \text { (i genap) } \\ 5 \mathrm{i}+1 & ; \mathrm{i}=5 \\ 5 \mathrm{i}+3 & ; 7 \leq \mathrm{i} \leq \mathrm{n} \text { (i ganjil) }\end{cases}$

$\mathrm{f}_{2}\left(\mathrm{v}_{\mathrm{i}, 5}\right)=\left\{\begin{array}{cl}32-11 \mathrm{i} & ; \mathrm{i}=0,1 \\ \mathrm{i}+15 & ; \mathrm{i}=2,3 \\ 5 \mathrm{i}+9 & ; 4 \leq \mathrm{i} \leq \mathrm{n}-1 \text { (i genap) } \\ 5 \mathrm{i}+3 & ; \mathrm{i}=5 \\ 5(\mathrm{i}+1) & ; 7 \leq \mathrm{i} \leq \mathrm{n} \text { (i ganjil) }\end{array}\right.$

Kasus $\mathrm{K}_{1, \mathrm{n}} \times \mathbf{P}_{6}$ :

\section{Subkasus 1 : $\mathbf{n}$ genap}

Akan ditunjukkan bahwa graf buku $\mathrm{K}_{1, \mathrm{n}} \times \mathrm{P}_{6}$ untuk $\mathrm{n} \geq 10$ adalah prime cordial dalam bentuk fungsi sebagai berikut: 


$$
\begin{aligned}
& \mathrm{f}_{1}\left(\mathrm{v}_{\mathrm{i}, 1}\right)=\left\{\begin{array}{cl}
2 & ; \mathrm{i}=0 \\
6 \mathrm{i}-5 & ; \mathrm{i}=1,2 \\
8 \mathrm{i}-18 & ; \mathrm{i}=3,5 \\
6 \mathrm{i}+1 & ; 4 \leq \mathrm{i} \leq \mathrm{n} \text { (i genap) } \\
6(\mathrm{i}-1) & ; 7 \leq \mathrm{i} \leq 11 \text { (i ganjil) } \\
6 \mathrm{i}-4 & ; 13 \leq \mathrm{i} \leq \mathrm{n}-1 \text { (i ganjil) }
\end{array}\right. \\
& \mathrm{f}_{1}\left(\mathrm{v}_{\mathrm{i}, 2}\right)=\left\{\begin{array}{cl}
4 & ; \mathrm{i}=0 \\
6 \mathrm{i}-1 & ; \mathrm{i}=1,2 \\
7 \mathrm{i}-11 & ; 3 \leq \mathrm{i} \leq 7 \text { (i ganjil) } \\
6 \mathrm{i}+3 & ; 4 \leq \mathrm{i} \leq \mathrm{n} \text { (i genap) } \\
6 \mathrm{i}-4 & ; \mathrm{i}=9,11 \\
6 \mathrm{i}-2 & ; 13 \leq \mathrm{i} \leq \mathrm{n}-1 \text { (i ganjil) }
\end{array}\right. \\
& \mathrm{f}_{1}\left(\mathrm{v}_{\mathrm{i}, 3}\right)=\left\{\begin{array}{cl}
8 & ; \mathrm{i}=0 \\
10 \mathrm{i}-7 & ; \mathrm{i}=1,2 \\
7 \mathrm{i}-9 & ; 3 \leq \mathrm{i} \leq 7 \text { (i ganjil) } \\
6 \mathrm{i}+5 & ; 4 \leq \mathrm{i} \leq \mathrm{n} \text { (i genap) } \\
6 \mathrm{i}-2 & ; \mathrm{i}=9 \\
6 \mathrm{i} & ; 11 \leq \mathrm{i} \leq \mathrm{n}-1 \text { (i ganjil) }
\end{array}\right. \\
& \mathrm{f}_{1}\left(\mathrm{v}_{\mathrm{i}, 4}\right)=\left\{\begin{array}{cl}
16 & ; \mathrm{i}=0 \\
8 \mathrm{i}+1 & ; \mathrm{i}=1,2 \\
7(\mathrm{i}-1) & ; 3 \leq \mathrm{i} \leq 7 \text { (i ganjil) } \\
6 \mathrm{i}+7 & ; 4 \leq \mathrm{i} \leq \mathrm{n}-2 \text { (i genap) } \\
6 \mathrm{i} & ; \mathrm{i}=9 \\
6 \mathrm{i}+2 & ; 11 \leq \mathrm{i} \leq \mathrm{n}-1 \text { (i ganjil) } \\
6(\mathrm{i}+1) & ; \mathrm{i}=\mathrm{n}
\end{array}\right. \\
& \mathrm{f}_{1}\left(\mathrm{v}_{\mathrm{i}, 5}\right)=\left\{\begin{array}{cl}
32 & ; \mathrm{i}=0 \\
4 \mathrm{i}+11 & ; \mathrm{i}=1,2 \\
6 \mathrm{i} & ; \mathrm{i}=3,5 \\
6 \mathrm{i}+9 & ; 4 \leq \mathrm{i} \leq \mathrm{n}-2 \text { (i genap) } \\
6 \mathrm{i}+2 & ; \mathrm{i}=7,9 \\
6 \mathrm{i}+4 & ; 11 \leq \mathrm{i} \leq \mathrm{n}-1 \text { (i ganjil) } \\
6 \mathrm{i}+4 & ; \mathrm{i}=\mathrm{n}
\end{array}\right. \\
& \mathrm{f}_{1}\left(\mathrm{v}_{\mathrm{i}, 6}\right)=\left\{\begin{array}{cl}
64 & ; \mathrm{i}=0 \\
\frac{43-\mathrm{i}}{2} & ; \mathrm{i}=1,3 \\
6 \mathrm{i}+11 & ; 2 \leq \mathrm{i} \leq \mathrm{n}-2 \text { (i genap) } \\
6 \mathrm{i}+4 & ; 5 \leq \mathrm{i} \leq 9 \text { (i ganjil) } \\
6(\mathrm{i}+1) & ; 11 \leq \mathrm{i} \leq \mathrm{n}-1 \text { (i genap) } \\
6 \mathrm{i}+2 & ; \mathrm{i}=\mathrm{n}
\end{array}\right.
\end{aligned}
$$

\section{Subkasus 2 : n ganjil}

Akan ditunjukkan bahwa graf buku $\mathrm{K}_{1, \mathrm{n}} \times \mathrm{P}_{6}$ untuk $\mathrm{n} \geq 11$ adalah prime cordial dalam bentuk fungsi sebagai berikut : 
$\mathrm{f}_{2}\left(\mathrm{v}_{\mathrm{i}, 1}\right)=\left\{\begin{array}{cl}2 & ; \mathrm{i}=0 \\ 6 \mathrm{i}-5 & ; \mathrm{i}=1,2 \\ 8 \mathrm{i}-18 & ; \mathrm{i}=3,5 \\ 6 \mathrm{i}+1 & ; 4 \leq \mathrm{i} \leq \mathrm{n}-1 \text { (i genap) } \\ 6(\mathrm{i}-1) & ; 7 \leq \mathrm{i} \leq 11 \text { (i ganjil) } \\ 6 \mathrm{i}-4 & ; 13 \leq \mathrm{i} \leq \mathrm{n} \text { (i ganjil) }\end{array}\right.$

$\mathrm{f}_{2}\left(\mathrm{v}_{\mathrm{i}, 2}\right)=\left\{\begin{array}{cl}4 & ; \mathrm{i}=0 \\ 6 \mathrm{i}-1 & ; \mathrm{i}=1,2 \\ 7 \mathrm{i}-11 & ; 3 \leq \mathrm{i} \leq 7 \text { (i ganjil) } \\ 6 \mathrm{i}+3 & ; 4 \leq \mathrm{i} \leq \mathrm{n}-1 \text { (i genap) } \\ 6 \mathrm{i}-4 & ; \mathrm{i}=9,11 \\ 6 \mathrm{i}-2 & ; 13 \leq \mathrm{i} \leq \mathrm{n} \text { (i ganjil) }\end{array}\right.$

$\mathrm{f}_{2}\left(\mathrm{v}_{\mathrm{i}, 3}\right)=\left\{\begin{array}{cl}8 & ; \mathrm{i}=0 \\ 10 \mathrm{i}-7 & ; \mathrm{i}=1,2 \\ 7 \mathrm{i}-9 & ; 3 \leq \mathrm{i} \leq 7 \text { (i ganjil) } \\ 6 \mathrm{i}+5 & ; 4 \leq \mathrm{i} \leq \mathrm{n}-1 \text { (i genap) } \\ 6 \mathrm{i}-2 & ; \mathrm{i}=9 \\ 6 \mathrm{i} & ; 11 \leq \mathrm{i} \leq \mathrm{n} \text { (i ganjil) }\end{array}\right.$

$\mathrm{f}_{2}\left(\mathrm{v}_{\mathrm{i}, 4}\right)=\left\{\begin{array}{cl}16 & ; \mathrm{i}=0 \\ 8 \mathrm{i}+1 & ; \mathrm{i}=1,2 \\ 7(\mathrm{i}-1) & ; 3 \leq \mathrm{i} \leq 7 \text { (i ganjil) } \\ 6 \mathrm{i}+7 & ; 4 \leq \mathrm{i} \leq \mathrm{n}-1 \text { (i genap) } \\ 6 \mathrm{i} & ; \mathrm{i}=9 \\ 6 \mathrm{i}+2 & ; 11 \leq \mathrm{i} \leq \mathrm{n} \text { (i ganjil) }\end{array}\right.$

$\mathrm{f}_{2}\left(\mathrm{v}_{\mathrm{i}, 5}\right)=\left\{\begin{array}{cl}32 & ; \mathrm{i}=0 \\ 4 \mathrm{i}+11 & ; \mathrm{i}=1,2 \\ 6 \mathrm{i} & ; \mathrm{i}=3,5 \\ 6 \mathrm{i}+9 & ; 4 \leq \mathrm{i} \leq \mathrm{n}-1 \text { (i genap) } \\ 6 \mathrm{i}+2 & ; \mathrm{i}=7,9 \\ 6 \mathrm{i}+4 & ; 11 \leq \mathrm{i} \leq \mathrm{n} \text { (i ganjil) }\end{array}\right.$

$\mathrm{f}_{2}\left(\mathrm{v}_{\mathrm{i}, 6}\right)=\left\{\begin{array}{cl}64 & ; \mathrm{i}=0 \\ \frac{43-\mathrm{i}}{2} & ; \mathrm{i}=1,3 \\ 6 \mathrm{i}+11 & ; 2 \leq \mathrm{i} \leq \mathrm{n}-1 \text { (i genap) } \\ 6 \mathrm{i}+4 & ; 5 \leq \mathrm{i} \leq 9 \text { (i ganjil) } \\ 6(\mathrm{i}+1) & ; 11 \leq \mathrm{i} \leq \mathrm{n} \text { (i ganjil) }\end{array}\right.$

Kasus $\mathrm{K}_{1, \mathbf{n}} \times \mathbf{P}_{\mathbf{7}}$ :

\section{Subkasus $1:$ n genap}

Akan ditunjukkan bahwa graf buku $\mathrm{K}_{1, \mathrm{n}} \times \mathrm{P}_{7}$ untuk $\mathrm{n} \geq 18$ adalah prime cordial dalam bentuk fungsi sebagai berikut :

$\mathrm{f}_{1}\left(\mathrm{v}_{\mathrm{i}, 1}\right)=\left\{\begin{array}{cl}2-\mathrm{i} & ; \mathrm{i}=0,1 \\ \mathrm{i}+3 & ; \mathrm{i}=2,3 \\ 29-\mathrm{i} & ; \mathrm{i}=4,5 \\ 7 \mathrm{i}+1 & ; 6 \leq \mathrm{i} \leq \mathrm{n} \text { (i genap) } \\ 7 \mathrm{i}-9 & ; \mathrm{i}=7,9 \\ 7(\mathrm{i}-1) & ; 11 \leq \mathrm{i} \leq 19 \text { (i ganjil) } \\ 7 \mathrm{i}-5 & ; 21 \leq \mathrm{i} \leq \mathrm{n}-1 \text { (i ganjil) }\end{array}\right.$ 
$\mathrm{f}_{1}\left(\mathrm{v}_{\mathrm{i}, 2}\right)=\left\{\begin{array}{cl}4 & ; \mathrm{i}=0 \\ 4 \mathrm{i}-1 & ; \mathrm{i}=1,2 \\ 8 \mathrm{i}-14 & ; 3 \leq \mathrm{i} \leq 7 \text { (i ganjil) } \\ 7 \mathrm{i}+1 & ; \mathrm{i}=4 \\ 7 \mathrm{i}+3 & ; 6 \leq \mathrm{i} \leq \mathrm{n} \text { (i genap) } \\ 6 \mathrm{i}+2 & ; \mathrm{i}=9 \\ 7 \mathrm{i}-5 & ; 11 \leq \mathrm{i} \leq 17 \text { (i ganjil) } \\ 7 \mathrm{i}-3 & ; 19 \leq \mathrm{i} \leq \mathrm{n}-1 \text { (i ganjil) }\end{array}\right.$
$\mathrm{f}_{1}\left(\mathrm{v}_{\mathrm{i}, 3}\right)=\left\{\begin{array}{cl}8 & ; \mathrm{i}=0 \\ 2 \mathrm{i}+7 & ; \mathrm{i}=1,2 \\ 8 \mathrm{i}-12 & ; 3 \leq \mathrm{i} \leq 7 \text { (i ganjil) } \\ 7 \mathrm{i}+3 & ; \mathrm{i}=4 \\ 7 \mathrm{i}+5 & ; 6 \leq \mathrm{i} \leq \mathrm{n} \text { (i genap) } \\ 6 \mathrm{i}+4 & ; \mathrm{i}=9 \\ 7 \mathrm{i}-3 & ; 11 \leq \mathrm{i} \leq 17 \text { (i ganjil) } \\ 7 \mathrm{i}-1 & ; 19 \leq \mathrm{i} \leq \mathrm{n}-1 \text { (i ganjil) }\end{array}\right)$
$\mathrm{f}_{1}\left(\mathrm{v}_{\mathrm{i}, 4}\right)=\left\{\begin{array}{cl}16 & ; \mathrm{i}=0 \\ 17-2 \mathrm{i} & ; \mathrm{i}=1,2 \\ 8 \mathrm{i}-10 & ; 3 \leq \mathrm{i} \leq 7 \text { (i ganjil) } \\ 7(\mathrm{i}+1) & ; 4 \leq \mathrm{i} \leq \mathrm{n}(\mathrm{i} \text { genap) } \\ 6(\mathrm{i}+1) & ; \mathrm{i}=9 \\ 7 \mathrm{i}-1 & ; 11 \leq \mathrm{i} \leq 17 \text { (i ganjil) } \\ 7 \mathrm{i}+1 & ; 19 \leq \mathrm{i} \leq \mathrm{n}-1 \text { (i ganjil) } \\ 32 & ; \mathrm{i}=0\end{array}\right.$

$\mathrm{f}_{1}\left(\mathrm{v}_{\mathrm{i}, 5}\right)=\left\{\begin{array}{cl}32 & ; \mathrm{i}=0 \\ 25-4 \mathrm{i} & ; \mathrm{i}=1,2 \\ 8 \mathrm{i}-6 & ; \mathrm{i}=3,5 \\ 7 \mathrm{i}+9 & ; 4 \leq \mathrm{i} \leq \mathrm{n}-2 \text { (i genap) } \\ 7 \mathrm{i}-1 & ; \mathrm{i}=7,9 \\ 7 \mathrm{i}+1 & ; 11 \leq \mathrm{i} \leq 17 \text { (i ganjil) } \\ 7 \mathrm{i}+3 & ; 19 \leq \mathrm{i} \leq \mathrm{n}-1 \text { (i ganjil) } \\ 7 \mathrm{i}+6 & ; \mathrm{i}=\mathrm{n}\end{array}\right.$

$\mathrm{f}_{1}\left(\mathrm{v}_{\mathrm{i}, 6}\right)=\left\{\begin{array}{cl}64 & ; \mathrm{i}=0 \\ 35-8 \mathrm{i} & ; \mathrm{i}=1,2 \\ 8 \mathrm{i}-4 & ; \mathrm{i}=3,5 \\ 7 \mathrm{i}+11 & ; 4 \leq \mathrm{i} \leq \mathrm{n}-2 \text { (i genap) } \\ 7 \mathrm{i}+1 & ; \mathrm{i}=7 \\ 7 \mathrm{i}+3 & ; 9 \leq \mathrm{i} \leq 17 \text { (i ganjil) } \\ 7 \mathrm{i}+5 & ; 19 \leq \mathrm{i} \leq \mathrm{n}-1 \text { (i ganjil) } \\ 7 \mathrm{i}+4 & ; \mathrm{i}=\mathrm{n}\end{array}\right.$

$\mathrm{f}_{1}\left(\mathrm{v}_{\mathrm{i}, 7}\right)=\left\{\begin{array}{cl}128 & ; \mathrm{i}=0 \\ 43-10 \mathrm{i} & ; \mathrm{i}=1,2 \\ 8 \mathrm{i}-2 & ; \mathrm{i}=3,5 \\ 7 \mathrm{i}+13 & ; 4 \leq \mathrm{i} \leq \mathrm{n}-2 \text { (i genap) } \\ 7 \mathrm{i}+3 & ; \mathrm{i}=7 \\ 7 \mathrm{i}+5 & ; 9 \leq \mathrm{i} \leq 17 \text { (i ganjil) } \\ 7(\mathrm{i}+1) & ; 19 \leq \mathrm{i} \leq \mathrm{n}-1 \text { (i ganjil) } \\ 7 \mathrm{i}+2 & ; \mathrm{i}=\mathrm{n}\end{array}\right.$

Subkasus 2 : n ganjil

Akan ditunjukkan bahwa graf buku $\mathrm{K}_{1, \mathrm{n}} \times \mathrm{P}_{7}$ untuk $\mathrm{n} \geq 19$ adalah prime cordial dalam bentuk fungsi sebagai berikut : 


$$
\begin{aligned}
& \mathrm{f}_{2}\left(\mathrm{v}_{\mathrm{i}, 1}\right)=\left\{\begin{array}{cl}
2-\mathrm{i} & ; \mathrm{i}=0,1 \\
9-\mathrm{i} & ; \mathrm{i}=2,3 \\
7 \mathrm{i}+1 & ; 4 \leq \mathrm{i} \leq \mathrm{n}-1 \text { (i genap) } \\
8(\mathrm{i}-2) & ; \mathrm{i}=5,7 \\
8 \mathrm{i}-18 & ; \mathrm{i}=9,11 \\
7(\mathrm{i}-1) & ; 13 \leq \mathrm{i} \leq 19 \text { (i ganjil) } \\
7 \mathrm{i}-5 & ; 21 \leq \mathrm{i} \leq \mathrm{n}(\mathrm{i} \text { ganjil) }
\end{array}\right. \\
& \mathrm{f}_{2}\left(\mathrm{v}_{\mathrm{i}, 2}\right)=\left\{\begin{array}{cl}
\text { i } & ; \mathrm{i}=0 \\
6 \mathrm{i}-1 & ; \mathrm{i}=1,2 \\
8 \mathrm{i}-14 & ; 3 \leq \mathrm{i} \leq 7 \text { (i ganjil) } \\
7 \mathrm{i}+3 & ; 4 \leq \mathrm{i} \leq \mathrm{n}-1 \text { (i genap) } \\
8(\mathrm{i}-2) & ; \mathrm{i}=9,11 \\
7 \mathrm{i}-5 & ; 13 \leq \mathrm{i} \leq 17 \text { (i ganjil) } \\
7 \mathrm{i}-3 & ; 19 \leq \mathrm{i} \leq \mathrm{n}(\mathrm{i} \text { ganjil) } \\
8 & ; \mathrm{i}=0
\end{array}\right. \\
& \mathrm{f}_{2}\left(\mathrm{v}_{\mathrm{i}, 7}\right)=\left\{\begin{array}{cl}
\left.\mathrm{f}_{\mathrm{i}, 3}\right)= \\
10 \mathrm{i}-7 & ; \mathrm{i}=1,2 \\
8 \mathrm{i}-12 & ; 3 \leq \mathrm{i} \leq 7 \text { (i ganjil) } \\
7 \mathrm{i}+5 & ; 4 \leq \mathrm{i} \leq \mathrm{n}-1 \text { (i genap) } \\
8 \mathrm{i}-14 & ; \mathrm{i}=9,11 \\
7 \mathrm{i}-3 & ; 13 \leq \mathrm{i} \leq 17 \text { (i ganjil) } \\
7 \mathrm{i}-1 & ; 19 \leq \mathrm{i} \leq \mathrm{n}(\mathrm{i} \text { ganjil) } \\
16 & ; \mathrm{i}=0 \\
7 \mathrm{i}+13 & ; 4 \leq \mathrm{i} \leq \mathrm{n}-1 \text { (i genap) } \\
8 \mathrm{i}-4 & ; \mathrm{i}=7,9 \\
7 \mathrm{i}+5 & ; 11 \leq \mathrm{i} \leq 17 \text { (i ganjil) } \\
7(\mathrm{i}+1) & ; 19 \leq \mathrm{i} \leq \mathrm{n}(\mathrm{i} \text { ganjil) }
\end{array}\right.
\end{aligned}
$$

\section{Teorema 2 :}

Graf matahari $C_{n} \odot \overline{K_{m}}$ adalah prime cordial untuk $n \geq 3$ dan $m \in\{2,3,5\}$. 


\section{Bukti :}

Kasus $\mathbf{C}_{\mathbf{n}} \odot \overline{\mathbf{K}_{\mathbf{2}}}$ :

Akan ditunjukkan bahwa graf matahari $\mathrm{C}_{\mathrm{n}} \odot \overline{\mathrm{K}_{2}}$ untuk $\mathrm{n} \geq 3$ adalah prime cordial dalam bentuk fungsi sebagai berikut :

$\mathrm{f}\left(\mathrm{v}_{\mathrm{i}}\right)= \begin{cases}3 \mathrm{i}-1 & ; \text { i ganjil } \\ 31-2 & ; \text { i genap }\end{cases}$

$f\left(v_{i, j}\right)=\left\{\begin{array}{cl}3 \mathrm{i}-2 & ; \mathrm{i} \text { ganjil, } \mathrm{j}=1 \\ 3 \mathrm{i}-1 & ; \mathrm{i} \text { genap, } \mathrm{j}=1 \\ 3 \mathrm{i} & ; 1 \leq \mathrm{i} \leq \mathrm{n}, \mathrm{j}=2\end{array}\right.$

Kasus $\mathbf{C}_{\mathbf{n}} \odot \overline{\mathbf{K}_{\mathbf{3}}}$ :

Akan ditunjukkan bahwa graf matahari $\mathrm{C}_{\mathrm{n}} \odot \overline{\mathrm{K}_{3}}$ untuk $\mathrm{n} \geq 3$ adalah prime cordial dalam bentuk fungsi sebagai berikut :

$\mathrm{f}\left(\mathrm{v}_{\mathrm{i}}\right)=4 \mathrm{i}-2 ; 1 \leq \mathrm{i} \leq \mathrm{n}$

$\mathrm{f}\left(\mathrm{v}_{\mathrm{i}, \mathrm{j}}\right)=\left\{\begin{array}{c}4 \mathrm{i}-3 ; 1 \leq \mathrm{i} \leq \mathrm{n}, \mathrm{j}=1 \\ 4 \mathrm{i}-1 ; 1 \leq \mathrm{i} \leq \mathrm{n}, \mathrm{j}=2 \\ 4 \mathrm{i} ; 1 \leq \mathrm{i} \leq \mathrm{n}, \mathrm{j}=3\end{array}\right.$

Kasus $\mathbf{C}_{\mathbf{n}} \odot \overline{\mathbf{K}_{\mathbf{5}}}$ :

Akan ditunjukkan bahwa graf matahari $\mathrm{C}_{\mathrm{n}} \odot \overline{\mathrm{K}_{5}}$ untuk $\mathrm{n} \geq 3$ adalah prime cordial dalam bentuk fungsi sebagai berikut :

$\mathrm{f}\left(\mathrm{v}_{\mathrm{i}}\right)=4 \mathrm{i}-2 ; 1 \leq \mathrm{i} \leq \mathrm{n}$.

$f\left(v_{i, j}\right)=\left\{\begin{array}{cc}6 i-5 & ; 1 \leq i \leq n, j=1 \\ 6 i-3 & ; 1 \leq i \leq n, j=2 \\ 6 i-2 & ; 1 \leq i \leq n, j=3 \\ 6 i-1 & ; 1 \leq i \leq n, j=4 \\ 6 i & ; 1 \leq i \leq n, j=5\end{array}\right.$

\section{KESIMPULAN}

Berdasarkan hasil penelitian yang telah dilakukan, dapat disimpulkan :

1. Graf buku $K_{1, n} \times P_{3}$ untuk setiap $n \geq 3$ adalah pelabelan prime cordial.

2. Graf buku $K_{1, n} \times P_{4}$ untuk setiap $n \geq 3$ adalah pelabelan prime cordial.

3. Graf buku $K_{1, n} \times P_{5}$ untuk setiap $n \geq 6$ adalah pelabelan prime cordial.

4. Graf buku $\mathrm{K}_{1, \mathrm{n}} \times \mathrm{P}_{6}$ untuk setiap $\mathrm{n} \geq 10$ adalah pelabelan prime cordial.

5. Graf buku $K_{1, n} \times P_{7}$ untuk setiap $n \geq 18$ adalah pelabelan prime cordial.

6. Graf matahari $\mathrm{C}_{\mathrm{n}} \odot \overline{\mathrm{K}_{2}}$ untuk setiap $\mathrm{n} \geq 3$ adalah pelabelan prime cordial.

7. Graf matahari $C_{n} \odot \overline{K_{3}}$ untuk setiap $n \geq 3$ adalah pelabelan prime cordial.

8. Graf matahari $\mathrm{C}_{\mathrm{n}} \odot \overline{\mathrm{K}_{5}}$ untuk setiap $\mathrm{n} \geq 3$ adalah pelabelan prime cordial. 


\section{DAFTAR PUSTAKA}

[1] Babujee, J.B., dan Shobana, L, 2009, Prime Cordial Labelings, Int. Review on Pure and Appl. Math.

[2] Cahayani, K.P., Soelistyo, R.H., dan Zaki, S, 2013, Pelabelan Cordial dan Graceful pada Arbitrary Supersubdivision Graf Path dan Star, Universitas Diponegoro, Semarang.

[3] Gallian, J.A, 2013, A Dynamic Survey of Graph Labeling, University of Minnesota Duluth, USA.

[4] Rohman, A.S, 2011, Pelabelan Cordial pada Graf Tangga $P_{n} \times P_{2}$ dan Graf Buku $K_{1, n} \times P_{2}$, Universitas Jember, Jember.

[5] Sundaram, M., Ponraj, R., dan Somasundram, S, 2005, Prime Cordial Labeling of Graphs, J. Indian Acad. Math.

[6] Vaidya, S.K., dan Vihol, P.L, 2010, Prime Cordial Labeling for Some Graphs, Modern Applied Science. 